\title{
Exploring the Effect of Food Intake and Physical Activity on Body Weight
}

\author{
Temiloluwa Prioleau, Yuqiang Heng, Ashok Veeraraghavan, Ashutosh Sabharwal
}

\begin{abstract}
Body weight is an important measurement parameter towards management of obesity, diabetes and overall health. Previous research and society at large has primarily focused on long-term fluctuation in body weight to describe and quantify weight gain or loss. Meanwhile, little emphasis has been placed on the potential benefit of monitoring shortterm body weight changes. Activities of daily living including food intake, excretion, and physical activities have a direct and measurable impact on short-term body weight in a day. Therefore, understanding the effect of these activities can provide insight towards development of a wearable system capable of detecting and quantifying daily intake, excretion and physical activities. In this study, body weight, intake, and physical activities were monitored intermittently for 7 - 8 hours on two separate days (sedentary and high activity). Results show that dietary intake always resulted in a positive change in body weight (mean error between measured body weight and amount of intake was $\pm 0.24 \mathrm{~kg}$ ). Meanwhile, high intensity physical activities (i.e. cardio) and excretion resulted in a negative change in body weight.
\end{abstract}

\section{INTRODUCTION}

Body weight is an important parameter to be monitored for prevention, delay and management of certain chronic conditions including obesity, diabetes and kidney failure. Research shows that frequent weight monitoring can be useful to support weight loss and management [1], [2]. There are a vast number of risk factors directly or indirectly related to unhealthy body weight. Overweight or obese conditions are primary risk factors for type 2 diabetes, which is then a risk factor for heart disease, stroke, blindness, kidney failure and lower-limb amputation [3], [4]. Long-term weight gain (or loss) often results from an energy imbalance in the body; when people eat or drink more calories than they burn, this can lead to weight gain and vice versa for weight loss [3]. Meanwhile, short-term weight gain (or loss) often results from voluntary activities in daily living. Daily fluctuations in body weight are common due to variations in body water, dietary habits and physical activity [5]. Therefore, short-term fluctuations in body weight can potentially be used towards objective quantification of the aforementioned activities of daily living (see Fig. 1).

Wearable systems such as FitBit@, Samsung Gear Fit ${ }^{\circledR}$ and Apple Watch ${ }^{\circledR}$ quantify physical activity in daily living using steps as the fundamental tracking parameter. These systems are relatively unobtrusive and estimate energy expenditure from ambulation and accelerometric data. On the

T. Prioleau, Y. Heng, A. Veeraraghavan and A. Sabharwal are with the Scalable Health Laboratory, School of Electrical and Computer Engineering, Rice University, Houston, TX, USA. (e-mail: to9@ rice.edu, ethan.heng@rice.edu, vashok@ rice.edu and ashu@ rice.edu).

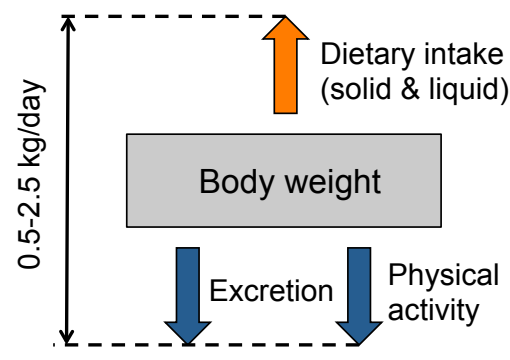

Fig. 1: Short-term body weight fluctuation

other hand, efforts towards objective quantification of energy intake are lagging. Food and drinks are the sole source of energy intake in regular living conditions. Therefore, the potential to automatically monitor intake in daily living is of high interest in the research community and society at large [6].

Amft [7] outlined food intake activities and bodily responses that can be sensed with wearable solutions for dietary monitoring. Majority of research literature on automatic food intake monitoring approaches the problem by tracking dietary activities including swallowing [8]-[10], chewing [11]-[13] and intake gesture [14], [15] as well as processing on meal images and videos such as in [16], [17]. Although, these activities provide information relevant for dietary monitoring, proposed on-body sensing locations which are primarily the neck/throat region, and in or around the ear can be potentially uncomfortable for a wearable system desired to be worn throughout the day. The recent paper [6] titled "unobtrusive and wearable systems for automatic dietary monitoring" provides a comprehensive review for understanding the state of this field. Unlike the common research approach aimed at detecting and monitoring activities that facilitate intake, this paper proposes the relevance of an additional parameter (body weight) which reflects the effect of intake and physical activity on the body.

Only a few papers in literature have attempted continuous monitoring of body weight in daily living [18], [19]. Sazonova et al. [18] focused on body weight prediction using a footwear-based system for estimation of daily energy expenditure. Whereas, Hellstrom et al. [19] explored carried load estimation during walking using a wearable weightsensing system. Both of these studies used a similar approach of incorporating force sensing resistors on a flexible insole for unobtrusive wearability.

This paper focuses on understanding the relationship between energy flux through dietary intake and physical activities, and short-term body weight fluctuation. Specific 
questions of interest are: 1) what is the relationship between intake and short-term body weight fluctuation?, 2) what is the relationship between physical activity and short-term body weight fluctation? The rest of the paper is organized as follows, section II describes the data collection method while section III describes data analysis and results as well as important considerations for developing a wearable insole for continuous body weight estimation. Lastly, section IV presents concluding remarks and future directions.

\section{Data Collection}

The data reported in this study was recorded from two subjects (male and female, ages 27 and 28, body mass index (BMI) 21 and 27.9) on two non-consecutive days. Body weight was collected using Ozeri Touch II - Total Body Scale (capacity: $200 \mathrm{~kg}$, graduation: $0.1 \mathrm{~kg}$ ), dietary intake was tracked with Ozeri Pronto Digital kitchen scale (capacity: 1 - $5 \mathrm{~kg}$, graduation: $0.001 \mathrm{~kg}$ ), while physical activity was tracked with the FitBit Charge. Weighing scales were placed on a hard and flat surface during weight measurements. Intermittent body weight and step count was monitored over a 7 - 8 hour period while subjects conducted regularly scheduled activities.

Day 1 included measurements of dietary intake (food $\&$ drinks) and body weight fluctuation on a lowactivity/sedentary day that consisted primarily of indoor activities, eating home-made meals/snacks and working on the computer. Meanwhile, day 2 included measurements of dietary intake and body weight fluctuation on a high activity day that consisted of aerobic/cardio exercise (running 3 miles at a park), eating lunch at a restaurant and running errands. Body weight and step counts were tracked intermittently through out the day ( $30-60$ mins) as well as immediately before and after activities expected to have a direct impact on weight, primarily intake, excretion and physical activity. Subjects wore the same clothing throughout the recording period in an effort not to introduce perceived body weight changes due to clothing or shoes. This study was approved by the Institutional Review Board of Rice University and all participants signed a written consent prior to the experiment.

\section{Data Analysis \& Results}

Graphs of continuous body weight measurements, change in step count and marked intake and excretion moments were plotted in MATLAB for each subject. Figure 2 shows the results from this observational study for subject 1 on day 1 - sedentary day (i.e. total step count $<2000$ steps/8hrs). It was observed that there was always an increase in the measured body weight due to intake events greater than the sensitivity of the scale $(0.1 \mathrm{~kg})$. It was also observed that excretion always showed up as a decrease in body weight, more noticable than the effect of physical activity on a low activity day. The body weight fluctuation range for subject 1 and 2 on day 1 - sedentary day was $\sim 1 \mathrm{~kg}(2.2 \mathrm{lbs})$.

Figure 3 shows the results for subject 1 on day 2 - high activity day (i.e. total step count $>9700$ steps/8hrs). Similar to day 1 , it was observed that there was always an increase in the measured body weight due to intake events and a decrease in body weight due to excretion. A decrease in body weight was also observed after high intensity physical activity (running 3 miles between 11:15 AM and 12:20 PM). An immediate post-exercise decrease in body weight of 0.45 $\mathrm{kg}(1 \mathrm{lb})$ and $0.72 \mathrm{~kg}(1.6 \mathrm{lbs})$ was observed for subject 1 and 2, respectively. An additional decrease in body weight of $(0.54 \mathrm{~kg}) 1.2 \mathrm{lbs}$ and $0.36 \mathrm{~kg}(0.8 \mathrm{lbs})$, not associated with excretion, was also observed within the following 3 hours post-exercise for subjects 1 and 2, respectively. The body weight fluctuation range for subject 1 and 2 on day 2 - high activity day was $1.4 \mathrm{~kg}$ (3 lbs) and $2 \mathrm{~kg}(4.4 \mathrm{lbs})$, respectively.

Figure 4 shows a graph of the amount of intake versus increase in body weight for both subject. A mean absolute error of $0.24 \mathrm{~kg}$ was observed across both subjects $(0.26 \mathrm{~kg}$ for subject 1 and $0.20 \mathrm{~kg}$ for subject 2) for the difference between amount of intake and increase in body weight. Therefore, the weight of intake was close but not equivalent to the observed increase in body weight. Some of this variance is a factor of the sensitivity difference between the food scale (graduation: $0.001 \mathrm{~kg}$ ) and the body scale (graduation: $0.1 \mathrm{~kg}$ ) used in this experiment. The positive correlation between amount of intake and body weight fluctuation indicates that continuous monitoring of body weight from a wearable system could potentially be used towards detecting moments of intake and for estimating the amount/volume consumed. Efforts towards dietary volume estimation have primarily been explored using image-based methods [20], [21]. A potential limitation of image-based methods for amount/volume estimation is the need for a reference object (i.e. fiducial marker) in the field of view during image capture for calibration purposes [21]. In addition, imagebased methods for dietary monitoring often rely on the user to initiate food image capture (i.e. user input is needed) and requires images of food selection before eating and leftovers after eating for consumed volume estimation [6].

\section{A. Considerations for Continuous Body Weight Measure- ment}

Continuous body weight measurements through out the day can potentially be beneficial for detecting moments of intake, excretion and physical activity as well as amount, volume, and intensity of these activities. Footwear based sensing platforms have the potential to enable ubiquitous monitoring in uncontrolled and free living conditions [22].

Figure 5 shows an example set-up for a wearable insole sensing system as well as the voltage measurements from each sensing element. FlexiForce ${ }^{\circledR}$ A201 by TekScan, Inc. was used to measure the force exerted on the insole. FlexiForce A201 supports standard force up to $4448 \mathrm{~N}$ (453.6 $\mathrm{kg}$ ). Its thin and flexible profile makes it easy to attach to an insole and does not cause discomfort for the wearer. We used an Arduino Uno as the microprocessor and attached 4 Tekscan FlexiForce A201 force sensitive resistors to the back of an insole, each connected in series with a $5.1 \mathrm{k} \Omega$ resistor to a power supply. We measure the force exerted on 


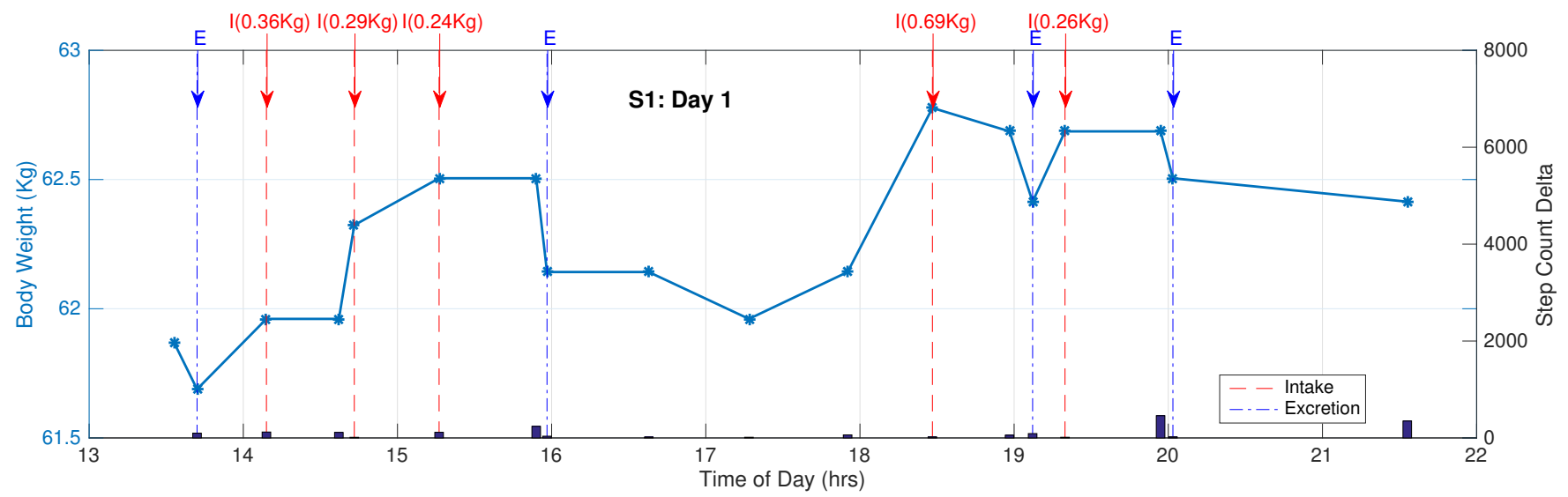

Fig. 2: Body weight in relation to intake, excretion and physical activity for Subject 1: Day 1 - Sedentary

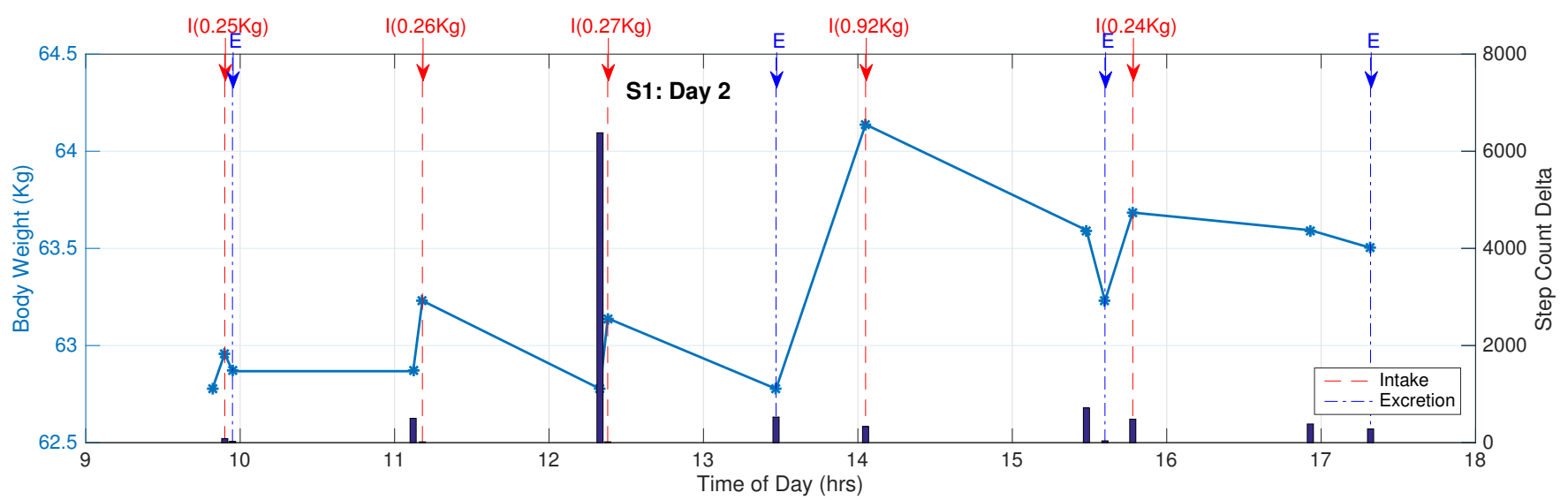

Fig. 3: Body weight in relation to intake, excretion and physical activity for Subject 1: Day 2 - High activity

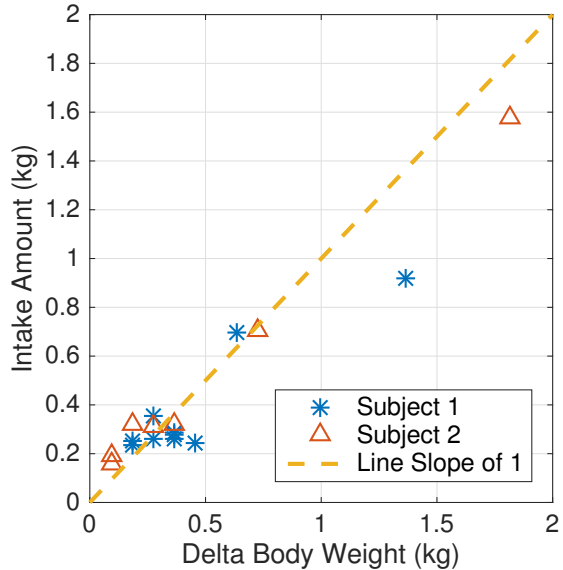

Fig. 4: Intake amount vs. increase in short-term body weight

the insole by measuring the voltage across the force sensitive resistors. The resistance of A201 is significantly bigger than $5.1 k \Omega$ when no force is applied and the measured voltage is close to $5 \mathrm{~V}$. When applied force increases, its resistance decreases and the measured voltage decreases as seen in Fig $5 b$.

From this experiment, we observed several considerations that should be taken into account towards the goal of

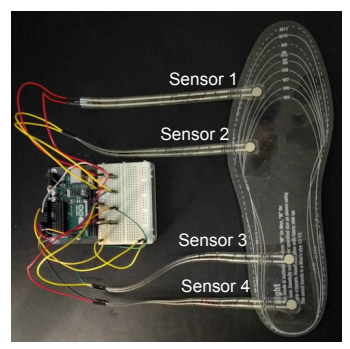

(a)

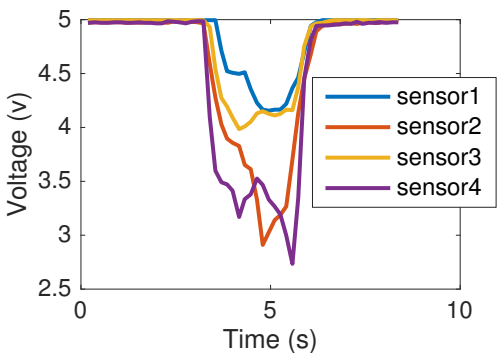

(b)
Fig. 5: Wearable insole sensing setup (a) Pressure sensing insole, (b) Pressure readings from each sensor

continuous body weight measurement from a wearable insole system. First and foremost, the effect of clothing, items carried and posture can manifest as a positive body weight change in the insole-recorded signal. Since this change will likely be similar and within expected variation range as dietary intake activities, a multimodal system is necessary to filter out such noise.

On another note, Hegde et al. [22] highlight the potential concern of drift of pressure sensing elements over time when used for continuous recording over long periods. This 


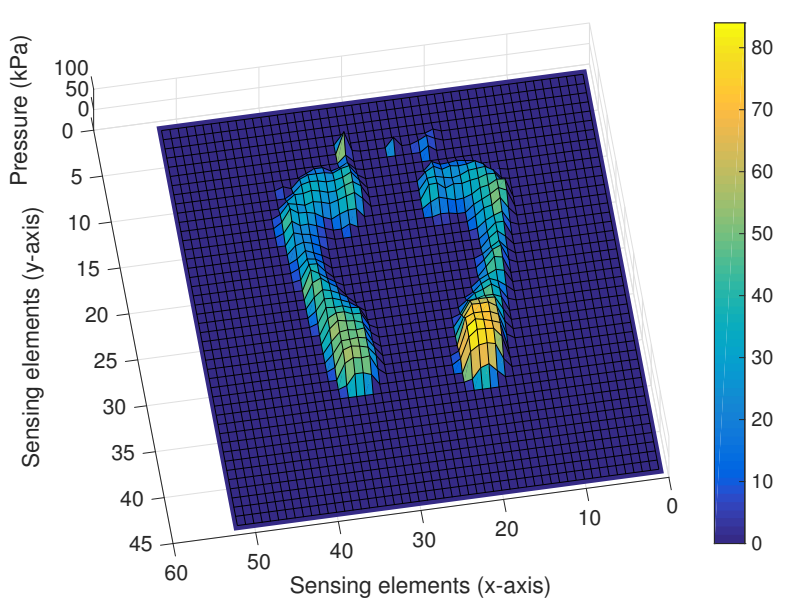

Fig. 6: Force mapping of the feet during standing posture

necessitates periodic calibrations to ensure preservation of accuracy. In addition, there is significant variation in force mapping across and even within individuals. Figure 6 shows the force mapping from the feet measured with MatScan ${ }^{\circledR}$ by Tekscan, Inc. during standing posture for subject 1 . Although, majority of the standing force is placed on the heel, metatarsal head, and hallux/big-toe, distribution of this force can and does vary even during standing. Capturing all of the distributed force (i.e. high spatial resolution) is important for body weight estimation from a wearable insole system. Inferred body weight is directly related to the total force underneath both feet and independent of the plantar pressure distribution across and within individuals. Limited spatial resolution in wearable systems could be part of the reason for high body weight estimation errors of $10.52 \mathrm{~kg}$ and $16.7 \mathrm{~kg}$ in [18] and [19], respectively.

$$
\text { Force }=\text { Mass } \times \text { Acceleration }
$$

Equation 1 shows that acceleration will have a direct effect on the relationship between the measured force and body weight (i.e. mass). Therefore, body weight estimations during detected periods of opportunistic standing as described in [18] is potentially a better approach than detecting periods of equipoise during walking for body weight estimation as described in [19].

\section{Conclusion And Future Work}

In this work, we have shown that short-term body weight is a potentially important and measurable parameter that can be used towards detecting and quantifying moments of intake, excretion and physical activities in daily living. We observed that intake of food and drinks amounts to a positive change in body weight, whereas excretion, sweating and high intensity physical activity amounts to a negative change in body weight. As expected, short-term weight fluctuated more (range $=1.4-2 \mathrm{~kg}$ ) on the high activity day than on the low activity day (range $=\sim 1 \mathrm{~kg}$ ). Also, low physical activity from a sedentary day did not have a measurable impact on short-term body weight fluctuation. In addition, we presented important considerations towards developing a wearable insole for continuous body weight estimation, namely, high spatial resolution on the sensing insole and body weight estimation during standing postures. Future work includes detecting moments of food intake and predicting intake volume throughout the day via a wearable insole system.

\section{REFERENCES}

[1] D. A. Levitsky et al., "Monitoring weight daily blocks the freshman weight gain: a model for combating the epidemic of obesity," Int. Jour of Obesity, vol. 30, no. 6, pp. 1003-1010, 2006.

[2] J. Vanwormer et al., "The impact of regular self-weighing on weight management: A systematic literature review,' Int J Behav Nutr Phys Act., vol. 5, pp. 1-10, 2008.

[3] U.S. Dept. of Health \& Human Services, "Overweight and obesity statistics," pp. 1-5, 2010.

[4] Centers for Disease Control and Prevention, "National diabetes statistics report: Estimates of diabetes and its burden in the United States,' 2014.

[5] S. B. Racette et al., "Influence of weekend lifestyle patterns on body weight," Obesity, vol. 16, no. 8, pp. 1826-30, 2008.

[6] T. Prioleau et al., "Unobtrusive and wearable systems for automatic dietary monitoring," IEEE Trans. on Biomed Eng. (Accepted), pp. 115, 2016.

[7] O. Amft and G. Tröster, "On-Body Sensing Solutions for Automatic Dietary Monitoring," Pervasive Computing, IEEE, pp. 62-70, 2009.

[8] O. Makeyev et al., "Automatic food intake detection based on swallowing sounds." Biomedical signal processing and control, vol. 7, no. 6, pp. 649-656, nov 2012.

[9] T. Olubanjo and M. Ghovanloo, "Real-time swallowing detection based on tracheal acoustics," in IEEE ICASSP, 2014.

[10] H. Kalantarian et al., "Monitoring eating habits using a piezoelectric sensor-based necklace," Comp. in Bio. \& Med., vol. 58, pp. 46-55, 2015.

[11] S. Päßler et al., "Food intake monitoring: an acoustical approach to automated food intake activity detection and classification of consumed food." Physiol. Measurement, vol. 33, pp. 1073-93, 2012.

[12] E. Sazonov and J. Fontana, "A sensor system for automatic detection of food intake through non-invasive monitoring of chewing." IEEE Sensors Journal, vol. 12, no. 5, pp. 1340-1348, 2012.

[13] T. Olubanjo et al., "Detecting food intake acoustic events in noisy recordings using template matching," in IEEE BHI, 2016.

[14] Y. Dong et al., "A new method for measuring meal intake in humans via automated wrist motion tracking," Applied Psychophysiology and Biofeedback, vol. 37, pp. 205-15, 2012.

[15] Y. Dong et al., "Detecting periods of eating during free-living by tracking wrist motion," IEEE J. of Biomed. \& Health Informatics, vol. 18, no. 4, pp. 1253-1260, 2014.

[16] M. Anthimopoulos et al., "Computer Vision-Based Carbohydrate Estimation for Type 1 Patients With Diabetes Using Smartphones," J. of Diabetes Science \& Technology, vol. 9, no. 3, pp. 507-515, 2015.

[17] C. K. Martin et al., "Measuring food intake with digital photography," Jour. of Human Nutrition \& Dietetics, vol. 27, pp. 72-81, 2014.

[18] N. A. Sazonova et al., "Prediction of bodyweight and energy expenditure using point pressure and foot acceleration measurements," Open Biomed Eng J., vol. 5, pp. 110-5, 2011.

[19] P. Hellstrom et al., "Wearable Weight Estimation System," Procedia Computer Science, vol. 64, pp. 146-152, 2015.

[20] C. Xu et al., "Image-based food volume estimation," Int. Workshop on Multimedia for Cooking \& Eating Activities (CEA), pp. 75-80, 2013.

[21] J. Chae et al., "Volume estimation using food specific shape templates in mobile image-based dietary assessment," in Proc SPIE Int Soc Opt Eng, 2011.

[22] N. Hegde et al., "A comparative review of footwear-based wearable systems," Electronics, vol. 5, no. 3, p. 48, 2016. 